\title{
AN IMPLANTABLE CAPACITIVE MICROMACHINED ULTRASONIC IMAGER PROBE FOR PHOTOACOUSTIC IMAGING
}

\author{
Xiaoyang Chengl, Jinguang Chen ${ }^{1}$, Sheng-Wen Huang ${ }^{2}$, Russell S. Witte ${ }^{3}$, and Pinyen Lin ${ }^{4}$ \\ ${ }^{1}$ Dept. of Electrical and Computer Engineering, \\ The University of New Mexico, Albuquerque, NM 87131 \\ ${ }^{2}$ Dept. of Biomedical Engineering, The University of Michigan, Ann Arbor MI 48109 \\ ${ }^{3}$ Dept. of Radiology, The Univ. of Arizona, Tucson, AZ 85721 \\ ${ }^{4}$ Infotonics Technology Center, Canandaigua, NY
}

\begin{abstract}
This paper reports the development of a miniature slab-shaped capacitive micromachined ultrasonic transducer (CMUT) array for photoacoustic imaging (PAI). As a minimally invasive imager, this device is capable of receiving relatively weak ultrasound signals that are difficult to access with non-invasive transducers and is useful for acquiring photoacoustic images of biological structures. This CMUT imager probe was fabricated using a two-layer polysilicon surface micromachining process, followed by a doublesided deep silicon etching process for shaping the silicon substrate into a thin probes. New CMUT structures were developed for this implantable imager probe, aiming at reducing the effective gap height and the driving voltage, as well as alleviating the charging trap effect. Photoacoustic imaging of the nerve cord of a lobster using this miniature CMUT array has been demonstrated.
\end{abstract}

\section{INTRODUCTION}

Capacitive Micro-machined Ultrasonic Transducers (CMUT) is considered as an alternative to the piezoelectric transducers for ultrasonic imaging. Extensive research on the fabrication and modeling of CMUT devices began in the early 1990s [1-2]. Compared to piezoelectric ultrasonic transducers, CMUT has the advantages of a broader acoustic bandwidth, feasibility of direct transducer-transistor integration, and a lower fabrication cost. Densely populated CMUT arrays can be manufactured using standard micro-fabrication techniques for high-frequency applications.

Photoacoustic imaging (PAI) [3-5] constructs images using ultrasounds generated by a target object which is under pulsed infrared illumination. The target object is flashed with a laser pulse, leading to optical absorption and thermoelastic expansion. This expansion generates ultrasound, which can be detected by an ultrasound transducer or an array of receivers to form images. Whereas traditional pulse-echo ultrasound imaging has low contrast in soft tissue due to similar acoustic impedances, PAI benefits from high optical contrast combined with excellent spatial resolution determined primarily by the ultrasound wavelength, approaching cellular resolution [6]. Contrast in PAI depends primarily on the optical wavelength and absorption spectrum of the tissue. Thus, PAI provides an appreciably higher contrast than pulse-echo ultrasonic imaging. Moreover, when the light source is tuned to the near infrared, PAI can be used to form an image well over a centimeter into tissue. For photoacoustc imaging of live human or animal tissue with red blood cells, hemoglobin provides significant help in boosting the contrast ratio. Hemoglobin has a very high optical contrast in the visible and infrared spectra. As a result, high-contrast imaging of blood containing structures in tissue such as tumors or blood vessel is one of the unique advantages of PAI. Compared to other techniques, PAI is a safe process that uses nonionizing radiation and fluences within standards set by ANSI and could provide 3D images with high resolution and contrast. In addition to viewing anatomical structure, photoacoustic imaging is capable of detecting composition of tissue and functional activities [5] of an organ based on blood-related infrared absorption rate difference and sensitivity of the optical spectrum of hemoglobin to oxygenation saturation [6].

Currently the vast majority of photoacoustic imaging experiments reported relied on use of single or an array of piezoelectric transducer for the ultrasound detection. CMUT [6-8] device is a promising alternative for PAI because of the broad acoustic bandwidth it provides. Fabricated using silicon-based integrated circuits technology, CMUT devices can be implemented into various 1-D [7], 2-D [7], or 3-D [8] arrays for PAI. In contrast to a regular laboratory-scale CMUT array [9], a miniature CMUT array [10] can be used in an invasive PAI system and would provide information not available from a non-invasive system. In this paper, the design of a miniature slab-shape CMUT array is reported, along with its application in photoacoustic imaging. Figure 1 illustrates the schematic of a PAI process using a miniature CMUT array.

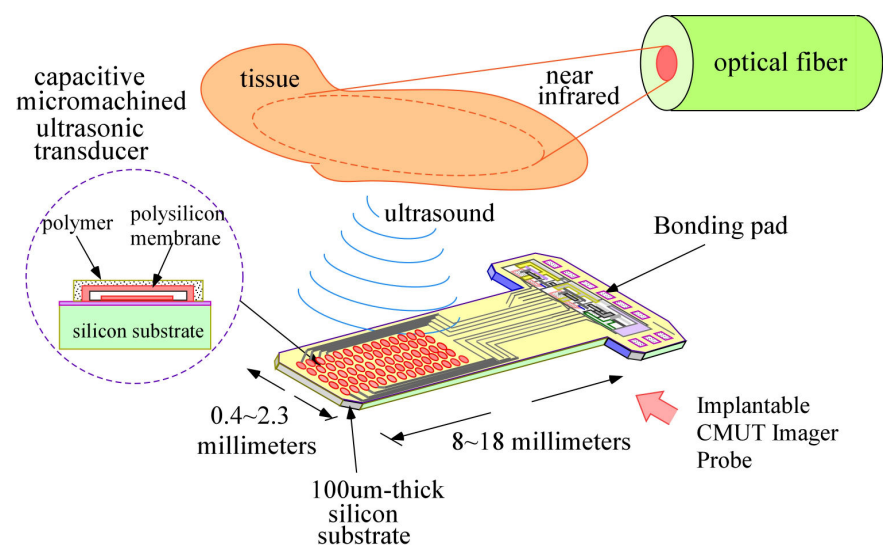

Figure 1: Schematic of the CMUT imager probe and its application in a photoacoustic imaging process.

\section{MINIATURE CMUT ARRAYS}

In many CMUT devices reported in the literature, a dielectric layer is embedded between the membrane and its counter electrodes to prevent electrode-membrane shorting. One of the problems associated with this embedded dielectric film is the charging problem. The charge stored in the dielectric-conductor 
interface will alter actuation voltages of the CMUT device. One of the solutions to alleviate the dielectric charging problem is to use a bipolar AC signal for driving the transducer [11] in a transmission process. However, even with application of these bipolar signals the charging effect cannot be completely eliminated. An embedded dielectric layer also increases the effective gap height of a CMUT, resulting in a higher driving voltage. For a CMUT with a dielectrics layer of thickness $x_{0}$, the effective gap is defined as [12]

$$
t_{\text {eff }}=S_{o}+\frac{x_{0}}{\varepsilon_{r}}
$$

Where $S_{0}$ is the gap distance, $\varepsilon_{r}$ is the relative dielectric coefficient of the material. The "pull-in" voltage for an ideal CMUT device with piston-like membrane is expressed as:

$$
V_{\text {collapse }}=\sqrt{\frac{2 k x}{\varepsilon_{0} A}} \times\left(S_{0}+\frac{x_{0}}{\varepsilon_{r}}-x\right)=\sqrt{\frac{8 k\left(S_{0}+x_{0} / \varepsilon_{r}\right)^{3}}{27 \varepsilon_{0} \pi a^{2}}}
$$

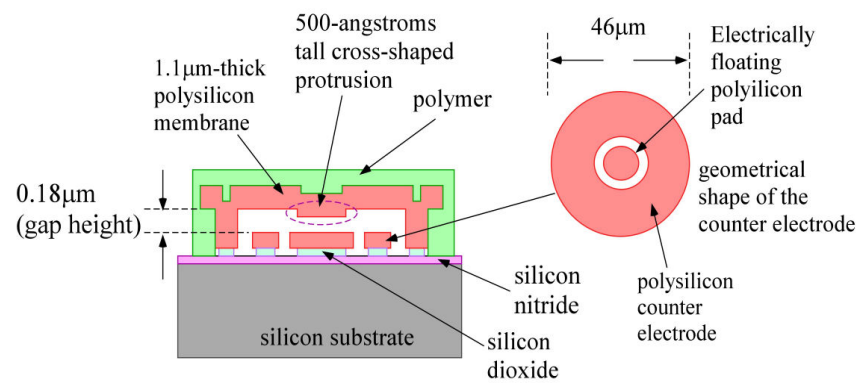

Figure 2: Poly-silicon capacitive micro-machined ultrasonic transducer with a circular-shaped buffering pad in the middle of the counter electrode.

A new CMUT structure was developed and used on this miniature PAI CMUT array as shown in Figure 2 to alleviate the aforementioned problems associated with an embedded dielectric layer. Instead of embedding a dielectric layer (either patterned or un-patterned) between the membrane and its counter electrode as commonly used for many CMUT devices, this new design used a pad-buffering structure for preventing shorting of the membrane to its counter electrode during ultrasound transduction. Because the dielectric layer is removed, the charging effect will be reduced. A circular-shaped $500 \AA$-high poly-silicon protrusion reaching out from the lower surface of the poly-silicon membrane works as the counter pad for the poly island. The polysilicon protrusion would land on the poly island when excessive electrical voltage or acoustic impingement is applied, preventing shorting of this device. Without an embedded dielectric layer, the effective gap distance [12] was reduced, at the cost of a smaller counter electrode area. For comparison, theoretical calculation was conducted based on the assumption that the electrodes act like parallel plates. The device parameters used in this calculation are listed in table 1. It was found that the island-insulated CMUT may be operated at a lower bias than its dielectric insulation counterpart under a comparable operation condition. Table 2 listed the calculated capacitance, collapse voltage, and effective gap height of $46 \mu$ m-diameter CMUT devices with an embedded dielectric layer or with a polysilicon island.
Table 1: CMUT Devices Parameters

\begin{tabular}{|l|l|}
\hline Cell diameter $(\mu \mathrm{m})$ & 46 \\
\hline Gap distance $(\mu \mathrm{m})$ & 0.3 \\
\hline Membrane thickness $(\mu \mathrm{m})$ & 1.0 \\
\hline Insulating layer thickness $(\mu \mathrm{m})$ & 0.12 \\
\hline Poly Island diameter $(\mu \mathrm{m})$ & 5.0 \\
\hline $\begin{array}{l}\text { Gap between the island and the ring- } \\
\text { shaped counter electrode }((\mu \mathrm{m})\end{array}$ & 1.5 \\
\hline
\end{tabular}

Table 2: The calculated results

\begin{tabular}{|l|l|l|}
\hline \multicolumn{1}{|c|}{ Parameters } & $\begin{array}{l}\text { CMUT with } \\
\text { dielectric } \\
\text { layer }\end{array}$ & $\begin{array}{l}\text { CMUT with } \\
\text { poly circular } \\
\text { protrusion }\end{array}$ \\
\hline Effective gap distance $(\mu m)$ & 0.22 & 0.2 \\
\hline Capacitance $(f F)$ & 70 & 65 \\
\hline Collapse voltages $(V)$ & 125 & 113 \\
\hline
\end{tabular}

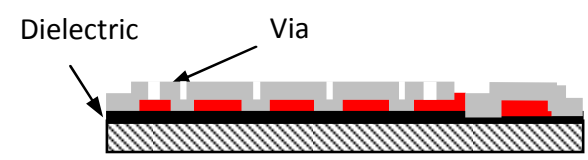

a) Thermal oxidation, LPCVD nitride/oxide deposition

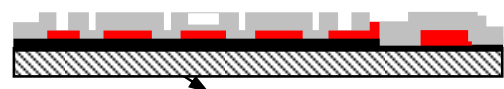

b) Sacrificial layer patterning to form the recess

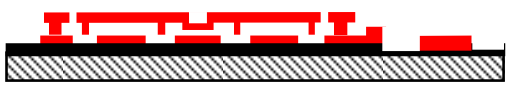

c) Poly1 deposition and sacrificial layer releasing

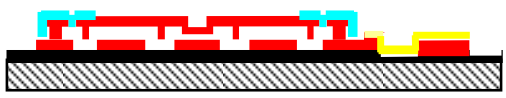

d) Sealing and metal sputtering

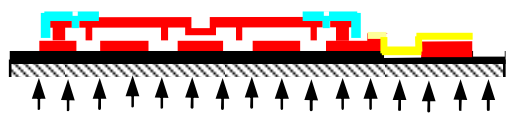

e) Backside etching, wafer front was protected with PR

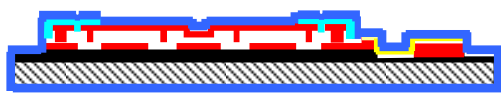

f) The parylene $\mathrm{C}$ sealing after wire bonding

$\begin{array}{lll}\text { substrate } & \text { Polysilicon } \\ \text { Parylene C } & \text { Metal } \\ \text { TEOS } & \text { PSG } \\ \text { Dielectric } & \end{array}$

Figure 3: the fabrication process flow

Two-layer-polysilicon micromachining process was employed to fabricate this device. The similar fabrication steps including surface micromachining and bulk micromachining can be found in 
[10] in detail. The big difference is that after the sacrificial layer (PSG) was deposited and the anchor was etched, the center of sacrificial layer was patterned and timely-control etched so that the circular protrusion could be formed during the Polyl deposition. The process flow was illustrated in Figure 3.

The SEM pictures of fabricated CMUT probes were shown in Figure 4. The array width of this 1D CMUT array varies from $1 \mathrm{~mm}$ to $4 \mathrm{~mm}$, depending on the frequency of the transducer. The length and thickness of the silicon substrate of the CMUT array are typically $1.2-2 \mathrm{~cm}$ and $100 \mu \mathrm{m}$, respectively. Such miniature CMUT arrays are suitable for implanting application or minimally invasive applications in surgery.

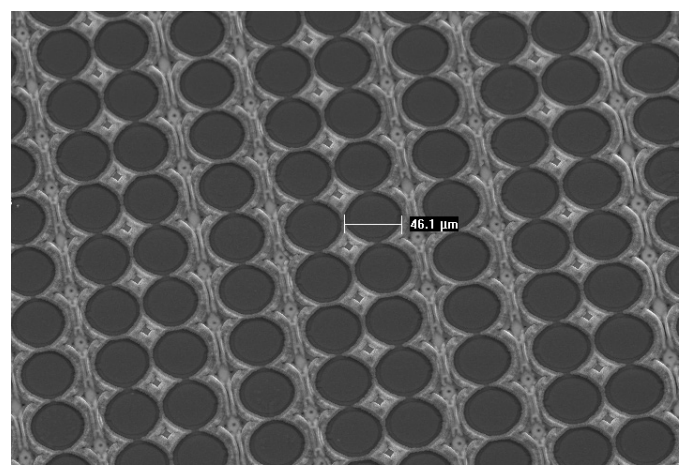

a)

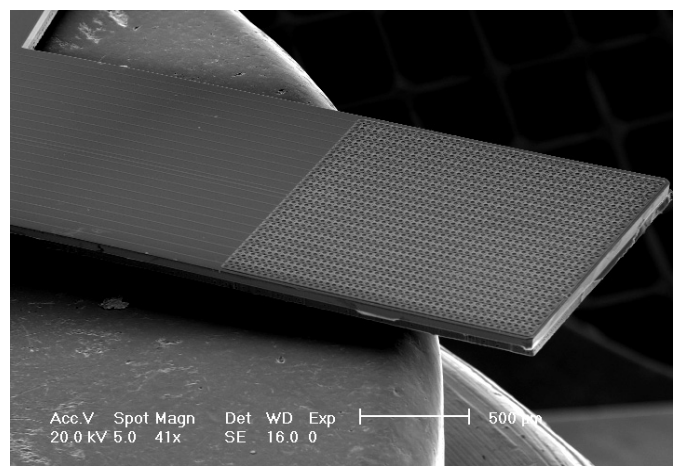

(b)

Figure 4: (a) SEM photograph of a two-dimensional CMUT array comprising 1058 transducers for photoacoustic imaging. (b) top view of the CMUT devices on this probe.

\section{DEVICE CHARACTERIZATION}

The CMUT array was tested both as a transmitter and a receiver in water. Figure 5 (a) \& (b) show the time- and frequencydomain ultrasound signal transmitted by a $46 \mu \mathrm{m}$-diameter CMUT and received by a commercial piezoelectric transducer; Figure 5 (c) \& (d) show the time- and frequency-domain ultrasound signal transmitted by a commercial piezoelectric ultrasound transducer and received by a $46 \mu \mathrm{m}$-diameter CMUT. The center frequency of the transmitting signal is about $5.2 \mathrm{MHz}$, which is close to the theoretical natural frequency $6 \mathrm{MHz}$. For CMUT working in the receiving mode, the center frequency is almost the same. The fractional bandwidth is around $98 \%$.

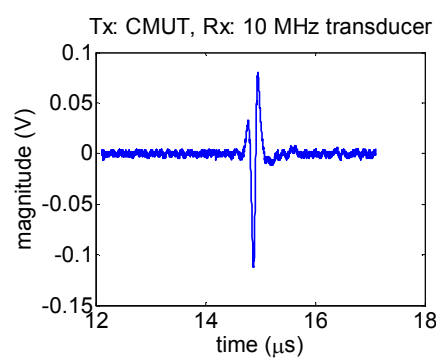

(a)

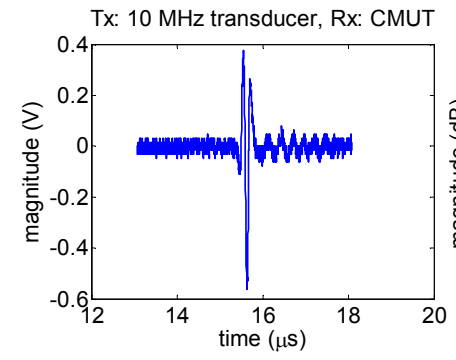

(c)

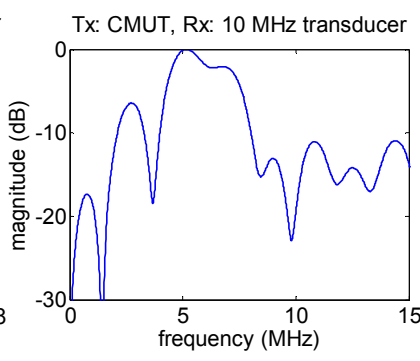

(b)

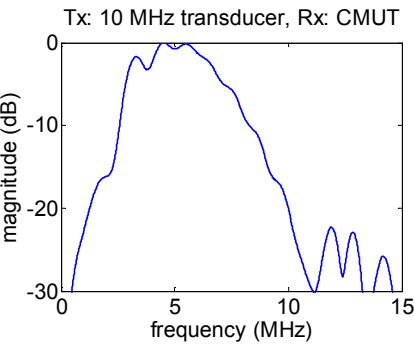

(d)
Figure 5: (a) \& (b) Time- and frequency-domain ultrasound signal in transmitting mode; (c) \& (d) time- and frequency-domain ultrasound signal in receiving mode.

Electrical testing was also performed to identify the charge trapping effect for this new design CMUT probe. For comparison, the previously designed CMUT with dielectric film covering the bottom electrode was biased at $50 \mathrm{Vdc}$ under receiving mode. It is observed that the received signal amplitude started to attenuate after $1 \mathrm{hr}$ due to charge trapping. In the test with this miniature slab-shaped CMUT, the received signal amplitude was not attenuated until the device continuously worked for 4 hours.

\section{PHOTOACOUSTIC IMAGING EXPERIMENT}

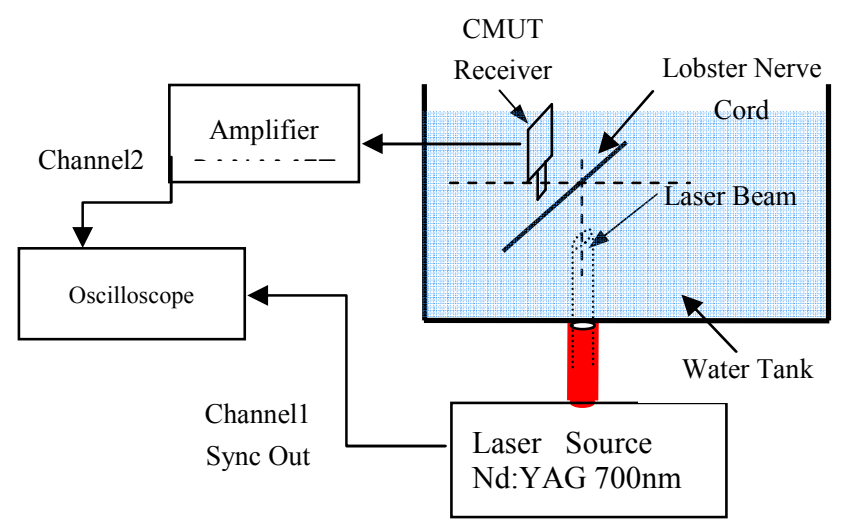

Figure 6: Schematic setup for the photoacoustic imaging system

In the photoacoustic imaging experiment, the miniature CMUT array was used as the receiving transducer for photoacoustic imaging of the lobster tissue. The schematic setup for the photoacoustic imaging is shown in Figure 6. The abdominal segment of the nerve cord of a lobster was excised, stained (with 
dye NK2761) and positioned in the testing water tank. A tunable laser source was used to generate laser pulses and create the approximate illumination pattern $(0.2 \times 2 \mathrm{~cm})$ on the nerve cord. The CMUT probe was placed perpendicularly to the laser beam axis and faced the nerve cord. The laser fired at PRR of $20 \mathrm{~Hz}$ with the wavelength at $700 \mathrm{~nm}$. The power intensity was approximately $20 \mathrm{~mJ} / \mathrm{cm}^{2}$. The signals were amplified with a $40 \mathrm{~dB}$ gain and digitized and then transferred to a computer. All post-processing was done using MATLAB. A synthetic aperture focusing technique (SAFT) was employed for imaging reconstruction. The reconstructed photoacoustic image of the stained nerve cord of a lobster is shown in Figure 7.

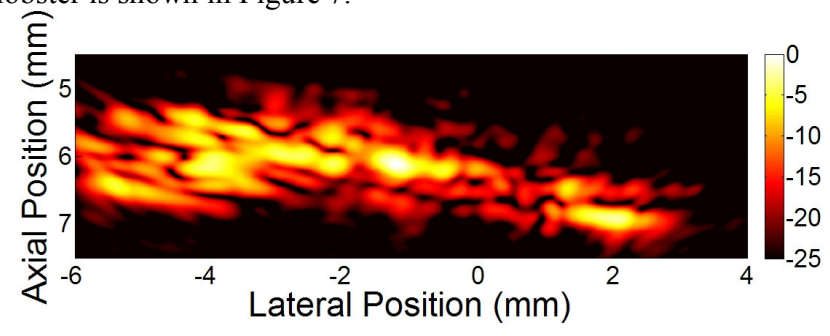

Figure 7: Photoacoustic image (dynamic range: $25 \mathrm{~dB}$ ) of the nerve cord of a lobster shot by the implantable CMUT probe. .

\section{CONCLUSION}

A miniature slab-shaped capacitive micromachined ultrasonic transducer (CMUT) imager and its application for photoacoustic imaging (PAI) were proposed in this research. The CMUT imager probe was fabricated using a two-layer polysilicon surface micromachining process. This image array reduced the effective gap. The experiment has shown that the charge trapping problem was alleviated. Photoacoustic imaging of the nerve cord of a lobster using this miniature CMUT array has also been successfully demonstrated.

\section{REFERENCES}

[1] M. I. Haller and B. T. Khuri-Yakub, "A surface micromachined electrostatic ultrasonic air transducer," in Proc. IEEE Ultrason. Symp., 1994, pp. 1241-1244.

[2] F. V. Hunt, "Electroacoustic: The analysis of transduction, and its historical background". New York, NY: Acoustical Society of America, 1982.

[3] J. E. Schneider, S. D. Bamforth, S. M. Grieve, K. Clarke, S. Bhatacharya, and S. Neubauer, "High resolution, highthroughput magnetic paragraph sign resonance imaging of mouse embryonic paragraph sign anatomy using a fast gradient-echo sequence.” Magma 16, 443-51 (2003).

[4] P. Mansfield, and P. G. Morris, NMR Imaging in Biomedicine, New York: Academic Press (1982).

[5] X. Wang, Y. Pang, G. Ku, X. Xie, G. Stoica, and L.-H. Wang, "Non-invasive laser-induced photoacoustic tomography for structural and functional imaging of the brain in vivo," Nature Biotechnology 21 (7), 803-806 (2003).

[6] M. Xu and L. V. Wang, "Photoacoustic imaging in biomedicine," Review of Scientific Instruments 77, 041101, pp. 1-22, 2006.
[7] Y. Huang, A. S. Ergun, M. H. Badi, and B. T. Khuri-Yakub, "Fabricating Capacitive Micromachined Ultrasonic Transducers with Wafer-Bonding Technology," IEEE Journal of Micromechanical Systems, vol. 12, no. 2, pp. 128137, 2003.

[8] J. Chen, X. Cheng, I.-M. Shen, J. Liu, P.-C. Li, and M. Wang, "A Monolithic Three-Dimensional Ultrasonic Transducer Array for Medical Imaging," IEEE Journal of Microelectromechanical Systems, vol. 16, no. 5, pp. 10151024, October 2007.

[9] I. O. Wygant, X. Zhuang, P. S. Kuo, D. T. Yeh, O. Oralkan, B. T. Khuri-Yakub, "Photoacoustic Imaging Using a TwoDimensional CMUT Array". 2005 IEEE Ultrasonics Symposium.

[10] J. Chen, X. Cheng, C. Chen, P. Li, J. Liu, and Y. Cheng, " A Capacitive Micromachined Ultrasonic Transducer Array for Minimally Invasive Medical Diagnosis " to be published in June/2008 issue of IEEE Journal of Microelectromechanical Systems.

[11] H. R. Shea, A. Gasparyan, H. B. Chan, S. Arney, R. E. Frahm, D. Lopez, S. Jin, R. P. McConnel, "Effects of electrical leakage currents on MEMS reliability and Performance". IEEE Trans. on Device and Materials Reliability, 2004, 4(2)198-207.

[12] M. I. Haller and B. T. Khuri-Yakub, "A surface micromachined electrostatic ultrasonic air transducer," in Proc. IEEE Ultrason. Symp., 1994, pp. 1241-1244. 